\title{
High Grade Serous Ovarian Cancer Presenting as Pseudo-Meigs Syndrome: A Case Report
}

\author{
Filipa Ferreira da Silva ${ }^{1 *}$, Luís Carreiro ${ }^{2}$, Inês Rolim ${ }^{3}$, Sara Belião ${ }^{4}$ and Henrique Nabais ${ }^{1}$ \\ ${ }^{1}$ Gynaecology Unit, Champalimaud Foundation, Portugal \\ ${ }^{2}$ Lung Unit, Champalimaud Foundation, Portugal
}

${ }^{3}$ Pathology Unit, Champalimaud Foundation, Portugal

${ }^{4}$ Radiology Unit, Champalimaud Foundation, Portugal

*Corresponding author: Filipa Ferreira da Silva, Medical Oncologist, Gynaecology Unit, Champalimaud Foundation, Lisbon, Portugal

\section{ARTICLE INFO}

Received: 崑 June 05, 2021

Published: 慧 June 21, 2021

Citation: Filipa Ferreira da Silva, Luís Carreiro, Inês Rolim, Sara Belião, Henrique Nabais. High Grade Serous Ovarian Cancer Presenting as Pseudo-Meigs Syndrome: A Case Report. Biomed J Sci \& Tech Res 36(4)-2021. BJSTR. MS.ID.005884.

Keywords: Pseudo Meigs Syndrome; Ovarian Cancer; Thoracic Staging

Abbreviations: OC: Ovarian Cancer; MS: Meigs Syndrome; Pseudo-MS: Pseudo Meigs Syndrome; HGSOC: High Grade Serous Ovarian Carcinoma; VATS: Video Assisted Thoracoscopic Surgery

\section{ABSTRACT}

Background: Classic Meigs and related syndromes are rare, but its recognition is important as it can mimic advanced malignant ovarian cancer. Contrary to what happens in advanced ovarian cancer, both pleural effusion and ascites resolve after surgery. Thoracic staging is therefore essential for the decision of primary treatment.

Case Presentation: 61-year-old female presenting with recurrent right pleural effusion without evidence of malignant cells. Investigation revealed moderate ascites and suspicious pelvic mass possibly originating from the ovary. After various attempts of pleural effusion drainage, the patient was submitted to video assisted thoracoscopic surgery for drainage, pleural biopsy and pleurodesis. The results showed no malignant cells and follow-up x-rays revealed residual pleural effusion. She underwent surgery with final pathology report consistent with stage IIB high grade serous ovarian carcinoma. No evidence of pleural effusion or ascites were documented on postoperative exams, and she started adjuvant chemotherapy.

Conclusion: Although rare, the various forms of Meigs Syndrome should be remembered in the presence of the triad: adnexal mass, pleural effusion, and ascites. Correct thoracic staging is essential for diagnosis and appropriate treatment, emphasizing the importance of a multidisciplinary evaluation of these cases.

\section{Background}

Ovarian cancer (OC) is the most lethal gynecological malignancy with an overall 5-year survival of 45\% [1]. Presence of pleural effusion, ascites, adnexal mass, and high levels of pretreatment CA125 are very suggestive of malignant ovarian tumor. Malignant pleural effusion must be confirmed as it is associated with worse prognosis and can influence the decision of primary treatment. Classic Meigs' syndrome (MS) was first described in 1937 by Meigs JW and Cass JW and consists of a benign solid ovarian fibroma with ascites and hydrothorax [2]. Both ascites and pleural effusion resolve after tumor removal. The prevalence of the syndrome is low but has important clinical implication as it mimics advanced malignant ovarian tumor presentation. In recent times other syndromes have emerged related to classic MS, namely malignant pseudo-Meigs'(Pseudo MS) which is referred to patients with pleural effusion, ascites and other benign or even malignant pelvic or abdominal tumors that lack evidence of peritoneal or pleural spread of the tumor (negative pleural and peritoneal fluid cytology and/or no malignant involvement in biopsy samples $[3,4]$. Although they are well-recognized syndromes, their etiology is not well defined and there are contradictory data regarding their 
specificities (site and pleural effusion characteristics) [5]. We herein report a case of pseudo-MS associated with high grade serous ovarian carcinoma (HGSOC) with high levels of CA125, ascites and recurrent pretreatment nonmalignant right pleural effusion.

\section{Case Presentation}

A61-year-old Caucasian female presented with a history of rapid progressive dyspnea and fatigue. She had a relevant past medical history of breast ductal carcinoma in situ diagnosed four years ago treated with breast-conserving surgery, adjuvant radiotherapy and tamoxifen; and past hysterectomy and right oophorectomy for a benign condition. A right pleural effusion was first documented at another institution and she underwent thoracic drainage and pleural biopsy. From what was reported, the aspirated fluid was compatible with an exudate, and both biopsy and liquid analysis were negative for malignancy. A diagnostic thoracic-abdominalpelvic CT scan was performed and showed right pleural effusion with atelectasis of right lower lobe, moderate ascites, and a pelvic mass of $13 \times 8 \mathrm{~cm}$ possibly with ovarian origin. For clarification, the patient performed an abdominal and pelvic MRI which highlighted the presence of intraperitoneal fluid and a predominantly solid mass of $13 \times 9 \times 11 \mathrm{~cm}$ originating from the left adnexal area (Figure 1). No suspicious lymph nodes were documented. The serum tumor marker levels of CEA, CA19-9 and CA15.3 were within range, but CA125 was elevated: $1498.0 \mathrm{U} / \mathrm{mL}$ (reference $<35 \mathrm{U} / \mathrm{mL}$ ). At our institution she was reevaluated in the Gynecology and Lung Units. Physical examination and ultrasonography revealed moderate right pleural effusion, and the patient was submitted to drainage with immediate removal of $1800 \mathrm{~mL}$ of Sero hemorrhagic pleural fluid. Once again pleural fluid was an exudate and malignant cells were not observed.

One week later, after maintaining pleural effusion even with a thoracic drain (drainage above 500mL day), she was submitted to Video-assisted thoracoscopic surgery (VATS) for pleural effusion drainage, pleural biopsy and pleurodesis (Figures 2 \& 3). No suspicious implants were documented during VATS. The results showed no malignant cells and follow up thoracic x-rays revealed residual pleural effusion. She then underwent surgery with extemporaneous examination of the adnexal tumor. Histopathological findings were consistent with HGSOC and cytoreductive surgery was performed: left anexectomy, segmental resection of sigmoid and omentectomy. The pathology report documented a FIGO stage IIB with extrinsic infiltration of sigmoid onto muscularis propria. The tumor demonstrated solid and papillary patterns, and the classic slit-like spaces. The cells showed typical nuclear pleomorphism, prominent nucleoli, and numerous mitotic figures. Immunohistochemistry confirmed nuclear expression of WT1, strong diffuse p16 immunoreactivity and abnormal pattern of p53 expression (mutation-type labelling) represented by strong diffuse staining in $>80 \%$ of tumor cells. The histological and immunohistochemical findings were of a HGSOC (Figure 4). Ascitic citology was negative for malignant cells. Postoperative CA125 was $43.3 \mathrm{U} / \mathrm{mL}$, she had no clinical evidence of ascites or pleural effusion and started adjuvant chemotherapy with carboplatin/paclitaxel (Figure 5). 


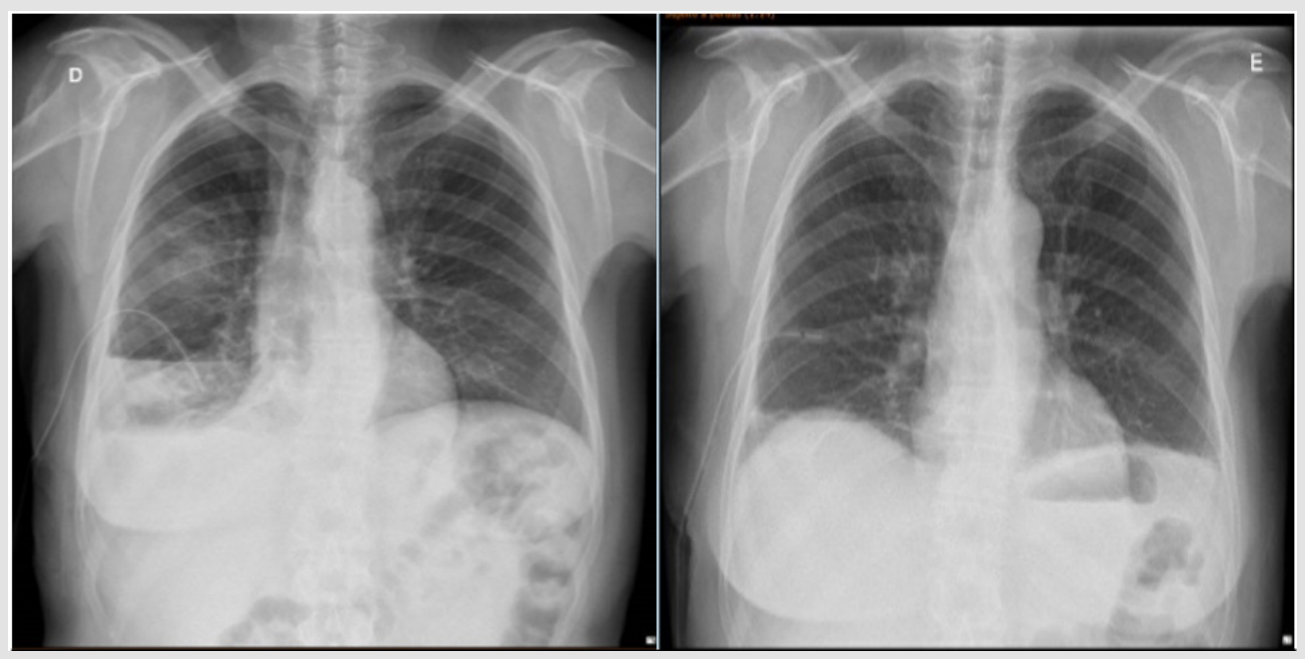

Figure 2: X-Rays Before VATS - 3 day evolution after chest drain insertion.

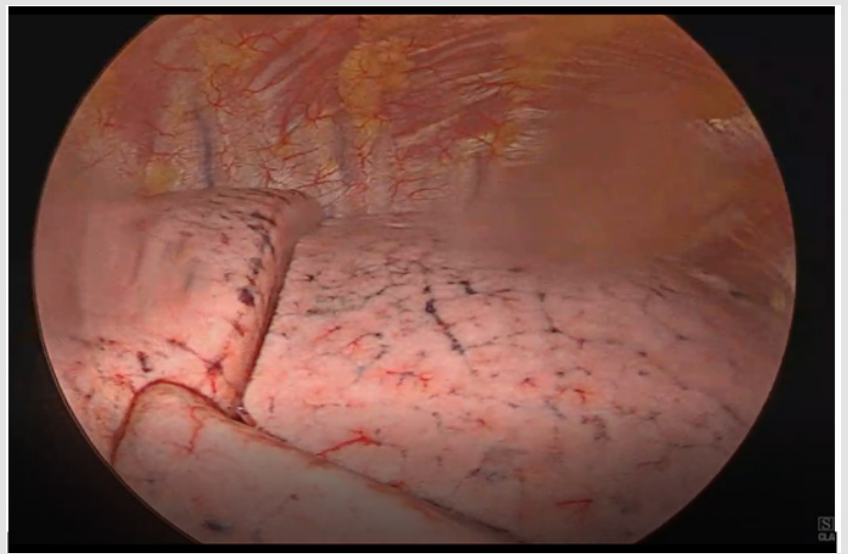

Figure 3: Image from VATS - Direct vision of lung and parietal pleural without implants
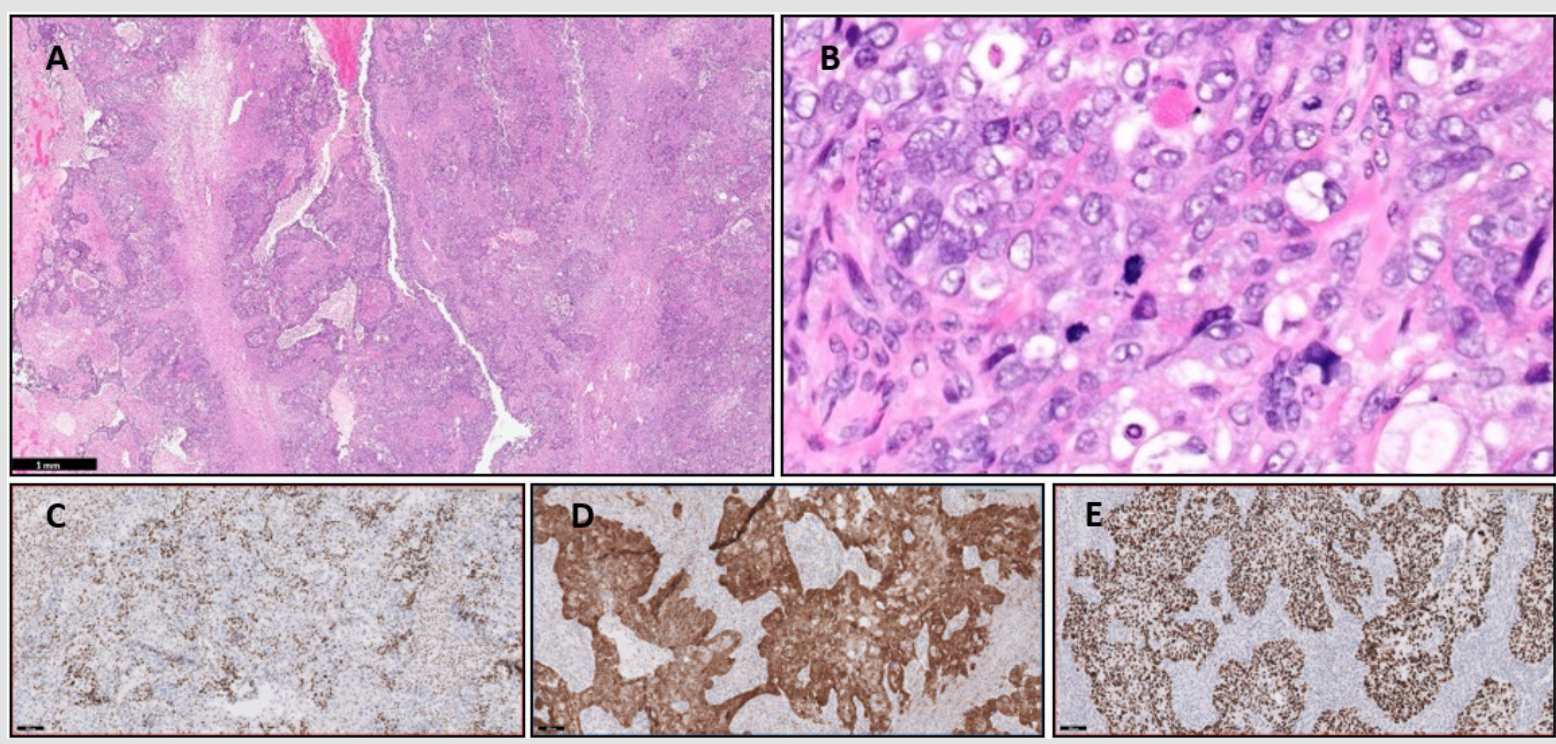

Figure 4: A-E. High-grade serous carcinoma. Epithelial neoplasm with solid and papillary patterns, and the classic slit-like spaces (A). The neoplastic cells show nuclear pleomorphism, prominent nucleoli, and mitotic figures (B). Immunohistochemistry confirmed WT1 (C), p16 (D) and p53 (E) positivity, the latter with a mutation-type labelling. 

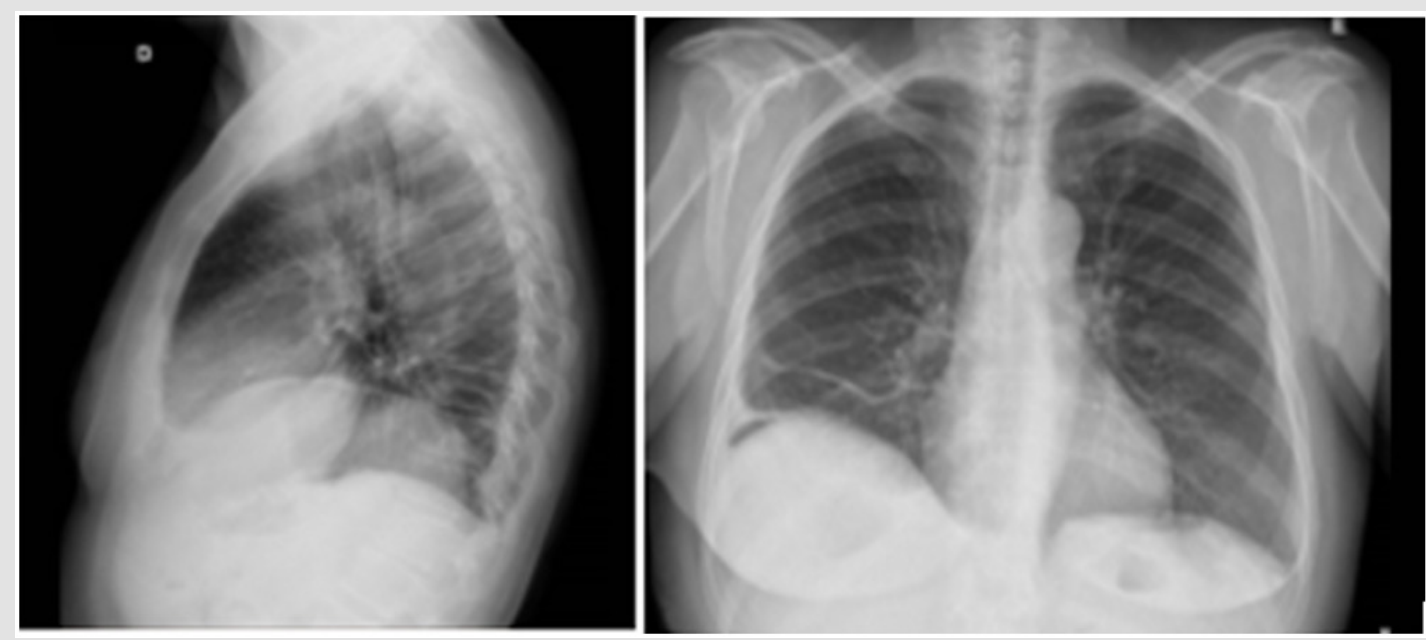

Figure 5: Five days post-op: Resolution of left pleural effusion.

\section{Discussion}

The patient's initial presentation pointed to a diagnosis of an advanced stage IVA ovarian carcinoma with the presence of ascites, recurrent pleural effusion, adrenal tumor and markedly elevated CA125. Despite its rarity and the fact that it is a diagnosis of exclusion, classic MS and its variants should be remembered as it can also lead to an increase in CA 125. Taking this into account the evaluation of pleural fluid is essential for proper staging and treatment. In this case the consecutive analysis of pleural effusion negative for malignant cells raised the hypothesis of Classic MS, which specifically refers to ovarian fibroma, or of pseudo-MS related to other benign or malignant abdominal and pelvic tumors. For clarification of the condition and in the face of recurrent pleural effusion the patient was submitted to VATS. We highlight the importance of this procedure in cases like this, considering the advantages it presents, in particular: visual evaluation of the pleura, direct tissue sampling (including pleural biopsy), and therapeutic intervention (in this case pleurodesis). Thus, we were able to say with greater certainty that there was no neoplastic thoracic involvement. After exclusion of thoracic involvement, the patient underwent primary surgery, during which the extemporaneous examination of the adnexal tumor revealed the diagnosis of malignancy (HGSOC) and cytoreductive surgery was performed.

Histopathological findings documented a FIGO stage IIB that presents better prognosis compared to the advanced stage initially suspected.

Both pleural effusion and ascites did not reappear after surgery, confirming the diagnosis of pseudo-MS. Malignant Pseudo-MS can be associated with other primary or secondary malignant pelvic or abdominal tumors. A systematic review of the literature published in 2015 [5] reported 75 cases of malignant Pseudo-MS, the majority with the diagnosis of primary ovarian tumor, with approximately two thirds presenting with right pleural effusion. Regarding pleural fluid characteristics, $60 \%$ were exudates and $61.4 \%$ had nonblood macroscopic appearance. Pseudo-Meigs' syndrome has already been reported associated with other tumors besides the ovary, with some reported cases referring to metastases of digestive tract and renal pelvis [6-8]. So in the presence of the triad (adnexal mass, pleural effusion and ascites) we must consider the differential diagnosis between benign and malignant abdominal and pelvic tumors that are not restricted to primary ovarian tumors.

\section{Conclusion}

Although rare, the various forms of MS should be remembered as a diagnostic hypothesis when we come across the triad of adnexal mass, pleural effusion and ascites. Correct thoracic staging is essential for the diagnosis and appropriate treatment. Finally, we should emphasize the importance of a multidisciplinary evaluation of these cases, with integration of teams of the gynecology, pulmonology, and radiology units.

\section{References}

1. Malvezzi M, Carioli G, Rodriguez T, Negri E, La Vecchia C (2016) Global trends and predictions in ovarian cancer mortality. Ann Oncol 27(11): 2017-2025.

2. Meigs JV, Cass JW (1937) Fibroma of the ovary with ascites and hydrothorax: With a report of seven cases. Am J Obstet Gynecol 33(2): 249-267.

3. Hartstein JA, Jacobs AJ, Deppe G, J Moshipur, C J Cohen, et al. (1980) Pseudo-Meigs syndrome with resulting papillary adenocarcinomas of the ovary and fallopian tube. Int J Gynaecol Obstet 18: 170-171.

4. Peparini N, Chirletti P (2009) Ovarian malignancies with cytologically negative pleural and peritoneal effusions: demons' or meigs' pseudosyndromes? Int J Surg Pathol 17(5): 396-397.

5. Krenke R, Maskey Warzechowska M, Korczynski P, Monika Zielinska Krawczyk, Joanna Klimiuk, et al. (2015) Pleural Effusion in Meigs' Syndrome-Transudate or Exudate?: Systematic Review of the Literature. Medicine (Baltimore) 94(49): e2114. 
6. Kyo K, Maema A, Shirakawa M, Nakamura T, Koda K, (2016) Pseudo Meigs' syndrome secondary to metachronous ovarian metastases from transverse colon cancer. World J Gastroenterol 22(18): 4604-4609.

7. Santos VM, Garcia CJFS, Lopes JWP, Marques Jr VCFM, Santos LAM (2013) A 74-year-old woman with peritoneal carcinomatosis: Diagnosis challenges. Brasília Med 50(2): 156-161.

\section{ISSN: 2574-1241}

DOI: 10.26717/BJSTR.2021.36.005884

Filipa Ferreira da Silva. Biomed J Sci \& Tech Res

(C) (P) This work is licensed under Creative

Submission Link: https://biomedres.us/submit-manuscript.php
8. Tessmer CS, Barcellos FC, Falchi LC (2005) Pseudo-Meigs' syndrome associated to renal pelvis tumor. International braz j urol : Official journal of the Brazilian Society of Urology 31(3): 256-258.

$\begin{array}{ll}\text { BIOMEDICAL } & \text { Assets of Publishing with us } \\ \text { RESEARCHES } & \text { - Global archiving of articles } \\ \text { - Immediate, unrestricted online access }\end{array}$

